\title{
Influence of Stern Shaft Inclination on the Cooling Performance of Water-Lubricated Bearing
}

\author{
Li ZOU ${ }^{a}$, Zheng Lin LIU \\ Wuhan University of Technology, Wuhan 430063, China
}

\begin{abstract}
The water film model of the marine water-lubricated stern bearing was established by FLUENT. The influence law of water flow rate on the cooling performance of water-lubricated bearing was studied in consideration of the stern shaft inclination. It will be helpful to improve the performance of marine water-lubricated stern bearing and both security and reliability of propulsion system. The simulation results show that the increase of cooling water flow ratein a certain range can effectively reduce bearing temperature. The bearing temperature rises sharply with thinning of water film thickness which is caused by the increase of inclination angle. Larger inclination angle can deteriorate the operating reliabilityof bearing.
\end{abstract}

\section{Introduction}

Many factors such as the cantilever action of propeller, shaft misalignment, bearing wear cancause the bending of marine stern shaft.The bending is either to increase the partial contact pressure of stern bearing or to result in the inclination of stern shaft, which will lead tothe boundary friction ofstern bearing, and the lubricating fluid cannot form hydrodynamic lubrication in this situation. What's more the increased friction factor will worsen the cooling performance of stern bearing.

The inclination of stern shaft both has the significant effect on the contact pressure of oil-lubricated and water-lubricatedbearings, especially a greater impact on the latter.Zhang et al. (2007) [1] analyzed the three-dimensional pressure distribution and temperature field of capillary-compensated water-lubricated hydrostatic journal bearings by solving the generalized Reynolds equation in the state of turbulent flow through the finite difference method, and the changes of the lubricant viscosity and density with temperature and a Li ZOU: 923309503@qq.com pressure were taken into account. Dai (2012) [2] analyzed the water-lubricated rubber bearings by using finite volume method, and revealed the influence law of both bearing structure parameters and operation condition on bearing temperature. Cheng (2012) [3] conducted the research on the water-lubricated bearing with the finite element software ADINA, discussed the influence of bearing structure and operation conditions on lubrication performance.

These studies as mentioned above were conducted in ideal state without considering the stern shaftinclination. Actually, due to the lining material of water-lubricated bearing is usually non-metallic one whose elastic modulus is much lower than metal material (such as Babbitt metal), so the stern shaftinclination will has greater influence on the lining deformation and the cooling performance of water-lubricated bearing. The main objective of this articleis to conduct a research on the cooling performance of water-lubricated bearingconsidering stern shaft inclination. According to the structure of water-lubricated 
bearing, a three-dimensional model of lubricating water film is established in GAMBIT. The influence of stern shaft inclination on the temperature distribution of water film is analyzed with finite volume method (FVM).

\section{Model}

\subsection{Bearing structure}

In this article,Thordon XL is selectedasthe lining material water-lubricated bearing. The material is of the superior resistance capacity such as impact, sediment and wear, but its limited operating temperature is $60{ }^{\circ} \mathrm{C}$. The water-lubricated bearing consists of bush and lining. The axial groove is used to improve the cooling and tribological performances of the bearing. Bearing's structure is shown in Fig. 1, geometric parameters are listed in Table 1 and physical properties of the material in Table 2 .

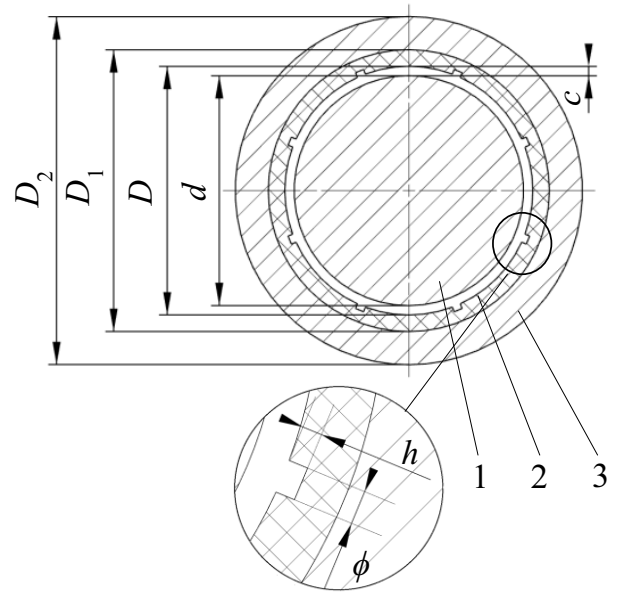

1-Sleeve 2-Lining 3-Bush

Figure 1. Section of shaft and bearing

Table 1.Structure parameters of bearing

\begin{tabular}{|c|c|}
\hline Parameters(units) & Values \\
\hline Shaft diameter(mm) & 298.4 \\
\hline Lining inner diameter(mm) & 300 \\
\hline Lining outer diameter(mm) & 340 \\
\hline Bearing outer diameter(mm) & 420 \\
\hline Bearing length $(\mathrm{mm})$ & 600 \\
\hline Groove depth $(\mathrm{mm})$ & 6 \\
\hline Bearing clearance $(\mathrm{mm})$ & 0.8 \\
\hline Groove width $\left({ }^{\circ}\right)$ & 3 \\
\hline
\end{tabular}

Table 2. Thermal properties of bearing materials

\begin{tabular}{|c|c|}
\hline Part(material) & Thermal conductivity \\
\hline Bush(Carbon steel) & $48 \mathrm{~W} \cdot(\mathrm{mK})^{-1}$ \\
\hline
\end{tabular}

\begin{tabular}{|c|c|}
\hline Lining(Thordon) & $0.25 \mathrm{~W} \cdot(\mathrm{mK})^{-1}$ \\
\hline Sleeve(Bronze) & $400 \mathrm{~W} \cdot(\mathrm{mK})^{-1}$ \\
\hline
\end{tabular}

\subsection{Calculation of bearing heat flux}

The friction heat source of the bearing consistsofthe friction heat generated by lubricant's shear force and cooling water heat. The friction heat calculation formula of the bearing:

$$
Q=\frac{2 \pi n}{60} M(1)
$$

Where $\boldsymbol{n}\left(\mathrm{r} \cdot \mathrm{min}^{-1}\right)$ is the rotational speed of stern shaft, $\boldsymbol{M}(\mathrm{N} \cdot \mathrm{m})$ is the frictional torque of stern bearing.

Stern bearing's frictional torque Mis composed of two parts. One part is generated by the lubricant, the other part is generated bysupporting load which isunrelated to velocity. When the stern shaft is in the state of hydrodynamic lubrication, the frictional torque produced by the lubricant should be considered. However, in actual operation, due to the influence of various factors (such as the cantilever of the propeller's gravity, propeller hydrodynamic etc.), the bearing supporting load is fluctuated, which will lead to the partial contact between stern shaft and bearing. At this time, the frictional torque generated by bearing supporting load will be dominant while that oflubricant is negligible.Therefore, the calculation formula of friction torquecan be expressed as:

$$
M=p D L \frac{d}{2} f(2)
$$

Where $\boldsymbol{p}(\mathrm{Pa})$ is the bearing supporting load, $D(\mathrm{~m})$ is the outer diameter of shaft, $L(\mathrm{~m})$ is the length of bearing, $d$ (m)is the inner diameter of bearing, $f$ is the equivalent friction coefficient derived from the relative experiment[4].

In this paper, the thermal conductivity of shaft sleeve $\left[400 \mathrm{~W} \cdot(\mathrm{mK})^{-1}\right]$ is much greater than that of lining $\left[0.25 \mathrm{~W} \cdot(\mathrm{mK})^{-1}\right]$, so that bearing friction heat can be totally applied on the outer surface of the shaft sleeve in the calculation. Considering energy loss in heat convection, the applied friction heat is actually $80 \%$ of totalheat[2].The bearing heat flux calculation formula:

$$
q=\frac{k Q}{\pi d L}(3)
$$

Where $k$ is the equivalent coefficient, $k=0.8$.

Parameters of bearing heat flux are listed in Table 3. 
Table 3. Parameters of friction heat calculation

\begin{tabular}{|c|c|}
\hline Parameters(units) & Values \\
\hline Bearing pressure $(\mathrm{MPa})$ & 0.4 \\
\hline Rotational speed $\left(\mathrm{r} \cdot \mathrm{min}^{-1}\right)$ & 300 \\
\hline Eccentricity & 0.9 \\
\hline Attitude angle $\left({ }^{\circ}\right)$ & 10 \\
\hline Equivalentfriction coefficient & 0.01 \\
\hline Bearing heat flux $\left(\mathrm{W} \cdot \mathrm{m}^{-2}\right)$ & 3374 \\
\hline
\end{tabular}

\subsection{Governing equation}

In this article, the governing equation used in FVM is:

$$
\frac{\partial(\rho \varphi)}{\partial t}+\operatorname{div}(\rho v \varphi)=\operatorname{div}\left(\Gamma_{\varphi} \operatorname{grad}(\varphi)\right)+S_{\varphi}(4)
$$

Where $\varphi$ is the variables, $\Gamma_{\varphi}$ is the corresponding diffusion coefficient of $\varphi, \quad S_{\varphi}$ is thecorrespondinggeneralized source of $\varphi, \operatorname{div}(\cdot)$ isthe divergence corresponding to a specific variable, $\operatorname{grad}(\cdot)$ is thegradient corresponding to a specific variable.

\subsection{CFD model, meshing and boundary definition}

Under the conditions of both low rotational speed and heavy load, the water film thickness of water-lubricated bearing can reach micron level.Therefore, the water film temperature can be regarded as the same as one of bearing inner surface in this situation. So the cooling performance of the bearing canbe evaluated according to the water film temperature.

Beforethe establishment of CFD water film model, the eccentricity, deflection angle and the inclinational angle of the stern shaft are set.The related parameters are listed in Table 3.

To reduce the quantity of mesh cells and to improve calculation precision, computational convergence, the hexahedralstructured mesh is adopted in the model. And because the axial length of model is much large than that of the radial direction, the mesh at in latter direction is refined.The finite volume model is shown in Fig. 2.

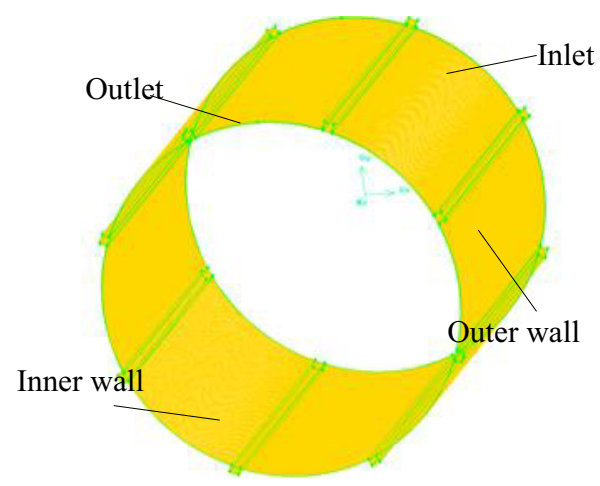

Figure2.CFD model of water film

In FLUENT, the boundary conditions of the model are set as follow:

(1)Inlet boundary is defined as velocity inlet;

(2)Outlet boundary is defined aspressure outlet;

(3) Outer wall boundary is defined as fix wall surface;

(4) Inner wall boundary is defined as rotating wall surface.

\section{Result and discussion}

China Classification Society (CCS)provides that the inclination angle of stern shaft shall not exceed $3.5 \times 10^{-4} \mathrm{rad}$. In this article, the cooling performance of stern bearing is discussed when the inclinational angle is $0,1 \times 10^{-4}, 2 \times 10^{-4}, 3 \times 10^{-4}, 4 \times 10^{-4} \mathrm{rad}$ respectively. The inclinational angle is shown in Fig. 3.

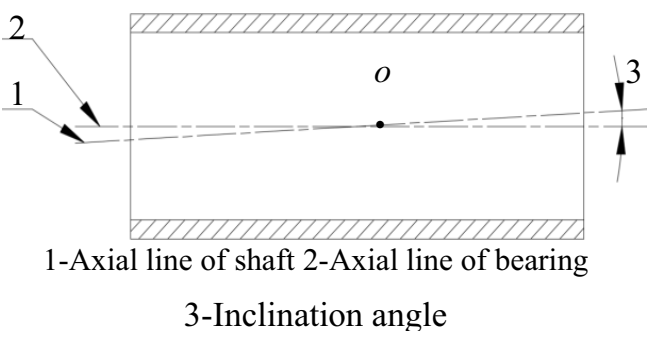

Figure3. Inclination angle

Under the operating pressure $0.4 \mathrm{MPa}$, rotational speed of shaft $300 \mathrm{r} \cdot \mathrm{min}^{-1}$, and bearing clearance $0.8 \mathrm{~mm}$, the temperature distributions at different water flow $\operatorname{speeds}(2$, $\left.4,6,8,10 \mathrm{~m} \cdot \mathrm{s}^{-1}\right)$ and inclinational angles $\left(0,1 \times 10^{-4}, 2 \times 10^{-4}\right.$, $\left.3 \times 10^{-4}, 4 \times 10^{-4} \mathrm{rad}\right)$ is analyzed, and the highest temperature and temperature distribution of water film are shown in Fig.4and Fig.5 respectively. 


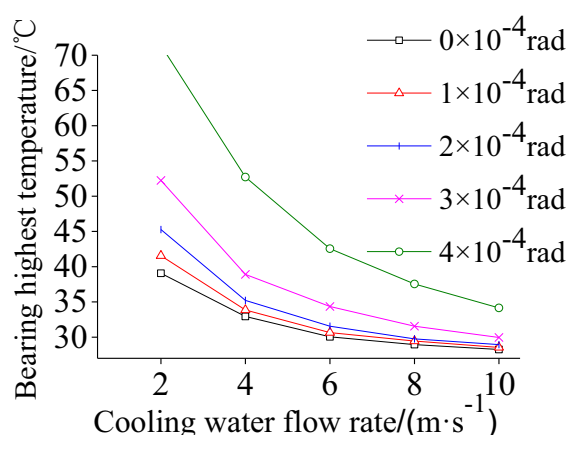

Figure4.Bearing highest temperature versus inclination angle for various flow rates

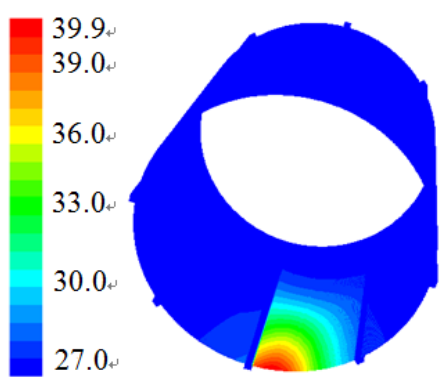

(a) Inclination angle is $0 \mathrm{rad}$,

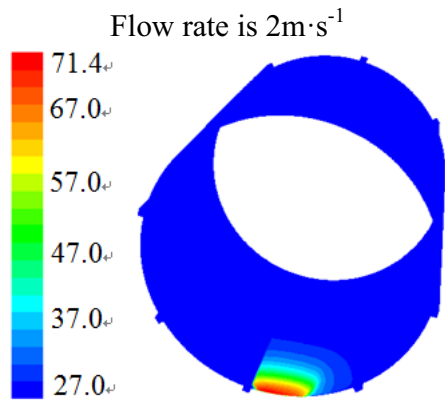

(b) Inclination angle is $4 \times 10^{-4} \mathrm{rad}$,

Flow rate is $2 \mathrm{~m} \cdot \mathrm{s}^{-1}$

Figure5.Bearing temperature distribution

It can be seen from Fig. 4, with the increase of cooling water flow rate, the highest temperatures of water film decline gradually. When the cooling water flow rate reaches $8 \mathrm{~m} \cdot \mathrm{s}^{-1}$, the water film temperature will be basically unchanged which indicates that thecooling water is sufficient to ensure the cooling effect at this time.The larger inclination angle is, the higher the water film temperature when the cooling water flow rate is constant. Contrasting the two curves whose inclination angles are $3 \times 10^{-4} \mathrm{rad}$ and $4 \times 10^{-4}$ radseparately, it can be seen that when the cooling water flow rate is increased from $2 \mathrm{~m} \cdot \mathrm{s}^{-1}$ to $10 \mathrm{~m} \cdot \mathrm{s}^{-1}$, the temperature rising rates are $48.7 \%, 46.9 \%$, $29.7 \%, 19.0 \%, 14.0 \%$ respectively. The results indicate that the flow rate is inversely proportional with the temperature rising rate.
It can be seen from Fig. 5, that the temperature at the bottom of water film is greatly changed and from the inlet to the outlet is gradually increased. The highest temperature region appears at the bottom of the outlet where the water film is the thinnest. The reason is that the thinner the water film is, the less the cooling water flow through the water film section and absorbed heat. And the more close to the outlet, the lower the cooling water flow rate is, the worse the cooling ability will be. As shown in Fig. 5(a) and 5(b), with the increase of inclination angle, the high temperature region of water film is closer to the outlet.

\section{Conclusion}

In this paper, the cooling performance of the water-lubricated bearing is studied with the finite volume method. The research conclusions as follows:

(1) The cooling water flow rate has a certain influence on the cooling performance of water-lubricated bearing.As the cooling water flow rate increases, the bearing temperature decreases. The minimum flow rate should be set to ensure the cooling effect.

(2) The inclination angle of stern shaft has a great influence on the cooling performance of water-lubricated bearing.As the inclination angle increases, the bearing temperature rises. Too large inclination angle will deteriorate the bearing working environment and result in excessive bearing temperature.

\section{Acknowledgment}

This work was financially supported by the project of Natural Science Foundation of China (No. 51379168), Key Natural Science Foundation of China (No. 51139005).

\section{References}

1. G. Zhang, X. Yuan, Lubrication Engineering, "A Theoretical Study on Three-dimensional Pressure Distributions and Temperature Field of Water Lubricated Hydrostatic Journal Bearings", No. 8, 4-7(2006)

2. Y. Dai, Wuhan University of Technology, "Influence of Water-lubricated Rubber Stern Tube Bearings Cooling Lubrication Performance on FLUENT", (2012)

3. Y. Cheng, Wuhan University of Technology, "Research on Lubricating Properties of Water Lubricated Stern Tube Bearings Based on TFSI", 
(2012)

4. Q. Yu, Jiangsu Ship, "Application of ThordonBearings on Ship", Vol. 17, No. 5, 26-30(2000) 